\title{
A ESPIRITUALIDADE E SEU IMPACTO NA SAÚDE
}

\author{
THE SPIRITUALITY AND IT'S IMPACT ON HEALTH
}

\author{
Aline Menegazzo Perse ${ }^{1 *}$, Andresa dos Santos Ferreira', Eduardo Dafllon Vinhosa Muniz ${ }^{1}$, Patrícia Alvarenga \\ Gonçalves Gomes Pereira ${ }^{1}$, Nathalia Fernanda da Silva Azevedo Rios ${ }^{2}$ \\ ${ }^{1}$ Acadêmicos de Medicina da Faculdade de Medicina de Campos- RJ \\ ${ }^{2}$ Médica Oncologista \\ *Autor de contato: alineperse@gmail.com
}

\section{RESUMO}

A espiritualidade poderia ser definida como uma propensão humana a buscar significado para a vida por meio de conceitos que transcendem o tangível: um sentido de conexão com algo maior que si próprio, que pode ou não incluir uma participação religiosa formal (Saad et al., 2001; Volcan, 2003). A espiritualidade e sua relação com a saúde tem se tornado claro paradigma a ser estabelecido na prática médica diária. A doença permanece como entidade de impacto amplo sobre aspectos de abordagem desde a fisiopatologia básica até sua complexa relação social, psíquica e econômica; é fundamental reconhecer que esses diversos aspectos estão correlacionados em múltipla interação.

Convém definir neste cenário que a religiosidade e a espiritualidade, apesar de relacionadas, não são claramente descritas como sinônimos. A religiosidade envolve sistematização de culto e doutrina compartilhados por um grupo. A espiritualidade está afeita a questões sobre o significado e o propósito da vida, com a crença em aspectos espiritualistas para justificar sua existência e significados (Saad et al., 2001; Powell et al., 2003).

A comprovação da utilização de aspectos distintos da espiritualidade e da religiosidade como suporte, terapêutica e determinação de desfechos positivos em diversas doenças tem constituído emblemático desafio para a ciência médica. Em se considerando as limitações éticas e de método, demonstra-se o quão dificultoso se faz mensurar e quantificar o impacto de experiências religiosas e espirituais pelos métodos científicos tradicionais.

Este artigo tem por objetivo apresentar de forma concisa as evidências recentes do papel da espiritualidade e da religiosidade em diversos campos da prática clínica diária.

Palavras chaves: espiritualidade, saúde, terapêutica, ciência.
ABSTRACT

A spirituality can be defined as a human propensity to seek meaning for life through concepts that transcend or tangible: a sense of connection with something greater that is yours, which may or may not include formal religious participation (Saad et al. , 2001; Volcan, 2003). Spirituality and its relationship with health have a clear paradigm to be established in daily medical practice. The disease remains an object of broad impact on aspects of approach from basic pathophysiology to its complex social, psychological and economic relationship; it is essential to recognize that these diverse aspects are correlated in interactive interaction.

It is important to define in this scenario that religiosity and spirituality, although related, are not clearly described as synonyms. Religiosity involves systematization of worship and doctrine shared by a group. Spirituality is prone to questions about the meaning and purpose of life, with a belief in spiritual aspects to justify its existence and meanings (Saad et al., 2001; Powell et al., 2003).

The proof of the use of different aspects of spirituality and religiosity as support, therapy and determination of positive outcomes in different diseases has been an emblematic challenge for medical science. Considering the ethical and method limitations, it is demonstrated how difficult it is to measure and quantify the impact of religious and spiritual experiences by traditional scientific methods.

This article aims to concisely present the recent evidence of the role of spirituality and religiosity in various fields of daily clinical practice.

Keywords-spirituality, health, therapeutics, science. 


\section{INTRODUÇÃO}

\section{Religiosidade e atividade imunológica}

A interleucina 6 (IL-6) tem se mostrado citocina fortemente correlata a reações agudas de estresse, doenças cardiovasculares, depressão, doenças osteomusculares, neoplasias e doença de Alzheimer (Lutgendorf et al., 2004).

Koenig et al. (1997) avaliaram a relação entre a prática de atividades religiosas e os níveis séricos de IL-6 e outros mediadores do sistema auto-imune e cascata inflamatória. Foram incluídos 1.718 pacientes com 65 ou mais anos de idade, e considerou-se como ponto de corte para IL- 6 de 5 $\mathrm{pg} / \mathrm{ml}$; a correlação à prática de serviços religiosos foi realizada de forma temporal em distintas entrevistas entre 1989 e 1992. Nos achados finais, comparados aos pacientes sem práticas religiosas freqüentes e controlando-se fatores de confusão, apenas a metade do grupo com práticas freqüentes

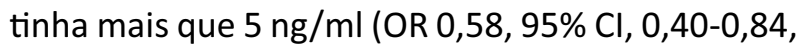
$p<0,005)$, além de menores taxas de marcadores de inflamação, como alfa-2 globulina, d-dímero, leucócitos polimorfonucleares e linfócitos.

Posteriormente, Harris et al. (1999a) descreveram que elevados níveis de IL-6, acima de $3,19 \mathrm{pg} / \mathrm{ml}$, são encontrados e mais estreitamente relacionados à mortalidade na população idosa que em grupos inferiores a $1,9 \mathrm{pg} / \mathrm{ml}$.

O mesmo grupo de autores, dessa vez capitaneado por Carrico et al. (2006), avaliou a relação entre práticas religiosas e níveis de cortisol urinário em 264 pacientes soropositivos para HIV, com o objetivo de determinar o impacto da espiritualidade sobre os níveis de cortisol e sua relação com sintomas de depressão; neste cenário, a prática de atividades religiosas esteve associada a menores taxas de cortisol urinário de 24 horas $(r=-$ $0,17, p<0,05)$ e sintomas de depressão.

\section{Religiosidade/espiritualidade e doença cardiovascular}

Goldbourt et al. (1993) avaliaram o impacto da religião ortodoxa na doença arterial coronária em 10.059 pessoas em Israel por cerca de 23 de seguimento, demonstrando associação não ajustada de RR de 0,69 ( $p=0,05)$ e após ajuste para fatores de confusão, como hábitos de vida, RR de $0,72(p=0,05)$. No entanto, certamente mais estudos longitudinais devem ser efetuados para a comprovação de sua relação com a morbidade cardiovascular.

\section{MÉTODOS}

Foram selecionados artigos no banco de dados Medline, Scielo, por meio dos unitermos: "religiosity", "religion", "spiritual" e "spirituality". Foram encontrados, inicialmente, cerca de 36 mil artigos; em segunda avaliação mais restrita, foram selecionados artigos de intervenções terapêuticas e revisões sistemáticas encontradas por meio de busca baseada no link "clinical queries" do referido sistema, totalizando 242 artigos. Os artigos foram avaliados por análise de método e determinação de limitações de desenho. Não sendo está uma metanálise, mas uma revisão descritiva apresenta-se a seguir as conclusões mais relevantes dos principais estudos e metanálises encontrados nesta revisão.

\section{Estudos em espiritualidade/religiosidade e saúde e seus vieses:}

Certamente, além de aspectos éticos e pessoais que podem promover vieses de análise e interpretação, os estudos envolvendo aspectos de religiosidade e espiritualidade merecem, como todo e qualquer outro objeto de investigação, a aplicação sistemática e rigorosa do método científico para sua validação, independentemente de posturas preconcebidas a favor ou contra eventuais resultados. Nesse contexto, há que se observar alguns frequentes vieses de apuração, desenho de estudo ou interpretação (Powell et al., 2003):

- Estudos ou cenários que não dispõem de adequado controle de potenciais fatores de confusão básicos, tais como idade, sexo e aspectos de etnia;

- Estudo de desenho cross-sectional ou transversal, considerado inadequado para determinar uma seqüência temporal de eventos, o que em geral é fundamental para uma adequada avaliação do nexo causal em estudos;

- Inadequada avaliação da mensuração da espiritualidade e/ou saúde física;

- Sem análises estatísticas apropriadas que impedem a adequada correlação entre achados;

- Relatos precoces em um mesmo estudo de coorte que apresenta distintos resultados com o passar do tempo em seu seguimento.

\section{RESULTADOS}

Strawbridge et al. (1997), em estudo de longo seguimento, avaliaram 6.928 pacientes, entre 16 e 94 anos, durante 28 anos de seguimento; os 
praticantes regulares de atividades religiosas tiveram menores taxas de mortalidade (razão de risco de 0,$64 ; 95 \%$ IC, 0,53-0,77). Esses resultados foram mais robustos em mulheres; em análise ajustada para antecedentes de doenças crônicas ou fatores de risco à saúde, não houve redução significativa do impacto. Durante o seguimento, os pacientes com práticas religiosas freqüentes interromperam o tabagismo, adotaram atividade física regular, aumentaram suporte social e mantiveram seu estado matrimonial.

Hummer et al. (1999) avaliaram dados do National Health Interview Survey (NHIS) em 21.204 casos e, entre estes, 2.216 óbitos, associando a freqüência de prática religiosa a aspectos sociodemográficos, de saúde e comportamento. Determinaram que pessoas que nunca tiveram ou que exerceram prática religiosa irregular apresentavam risco de óbito 1,87 vez maior comparadas àquelas com prática de pelo menos uma vez por semana. Tal associação se traduziu em diferença de cerca de até sete anos adicionais, na expectativa de vida entre os grupos.

Jaffe et al. (2005) avaliaram pacientes aderentes a práticas religiosas ou habitando áreas consideradas afiliadas a práticas religiosas em Israel. Foram analisados 141.683 indivíduos com idades de 45 a 89 anos, vivendo em 882 áreas distintas; 29.709 óbitos foram reportados em um seguimento médio de 9,5 anos. Homens e mulheres vivendo em áreas próximas ou afiliadas a práticas religiosas tiveram menores taxas de mortalidade (OR [homens] $=0,75 ; 95 \%$ IC, 0,67-0,84; OR [mulheres] $=0,86 ; 95 \% I C, 0,67-0,96)$.

Partindo desse cenário delimitado, em um estudo prospectivo, Lutgendorf et al. (2004) avaliaram a relação entre frequência de prática religiosa, níveis séricos de IL-6 e mortalidade em 557 adultos idosos. A prática de atividades religiosas de, pelo menos, uma vez por semana foi determinada como significativo preditor de menor mortalidade em 12 anos de seguimento e da menor elevação dos níveis de IL-6 (> 3,19 pg/ml): os achados do estudo demonstraram taxa de mortalidade de $0,32(95 \% \mathrm{IC}=0,15,0,72 ; p<0,01)$ e razão de chances (OR) para elevação de IL-6, 0,34 $(95 \% \mathrm{Cl}=0,16,0,73, \mathrm{p}<0,01$ em pacientes com práticas religiosas semanais, comparados àqueles que nunca frequentavam ou exerciam práticas religiosas. Os resultados foram analisados em regressão logística multivariada. A despeito dos resultados, há limitações do estudo quanto à mensuração isolada do envolvimento religioso do paciente e à frequência de prática e dados privados, como religiosidade intrínseca. No entanto, houve correlação linear entre o efeito da mortalidade entre os pacientes que nunca tinham práticas religiosas em relação àqueles que tiveram práticas de pelo menos uma a duas vezes por mês.

Ironson et al. (2006) avaliaram os efeitos de mudanças na religiosidade e na espiritualidade após o diagnóstico de soro positividade para o HIV e suas consequências sobre as dosagens de CD4 e carga viral em até quatro anos de seguimento; em 100 pacientes avaliados, $45 \%$ demonstraram aumento de práticas religiosas, $42 \%$ não alteraram suas práticas e $13 \%$ as reduziram. Utilizando um modelo de regressão linear, avaliou-se a associação de efeitos entre a prática religiosa, CD-4 e carga viral: independentemente do tipo de prática religiosa, quadro inicial da doença, medicações em uso, idade, sexo, etnia, educação, hábitos de vida, depressão e suporte social, a mudança na prática de atividades religiosas foi fator predito independente para redução da carga viral e aumento dos valores de CD4.

O mesmo grupo de autores, dessa vez capitaneado por Carrico et al. (2006), avaliou a relação entre práticas religiosas e níveis de cortisol urinário em 264 pacientes soropositivos para HIV, com o objetivo de determinar o impacto da espiritualidade sobre os níveis de cortisol e sua relação com sintomas de depressão; neste cenário, a prática de atividades religiosas esteve associada a menores taxas de cortisol urinário de 24 horas $(r=-$ $0,17, p<0,05)$ e sintomas de depressão.

\section{CONSIDERAÇÕES FINAIS}

A influência da religiosidade/espiritualidade tem demonstrado potencial impacto sobre a saúde física, definindo-se como possível fator de prevenção ao desenvolvimento de doenças, na população previamente sadia, e eventual redução de óbito ou impacto de diversas doenças. As evidências têm-se direcionado de forma mais robusta e consistente para o cenário de prevenção; estudos independentes, em sua maioria de grande número de voluntários e representativos da população, determinaram que a prática regular de atividades religiosas tem reduzido o risco de óbito em cerca de $30 \%$ e após ajustes para fatores de confusão, em até 
$25 \%$.

Estudos mecanísticos tentando avaliar qual a relação entre redução de mortalidade e práticas religiosas têm enfatizado o possível incentivo que essas práticas oferecem a hábitos de vida saudável, suporte social, menores taxas de estresse e depressão. Atitudes assistenciais voluntárias ou participação em congregações têm demonstrado associação com redução de mortalidade, provendo suporte e significado de vida, emotividade de aspecto positivo ou ausência de emoções consideradas de aspecto negativo; certamente existem dúvidas sobre se esses aspectos são mais relevantes em grupos específicos, tais como no de sexo feminino, com menor suporte socioeconômico e menores níveis de educação.

Também em contraste com tais evidências, não há clara correlação entre o grau de profundidade ou envolvimento em práticas religiosas e a proteção a eventos, exceto por análises de subgrupos em curva post hoc. O desenvolvimento de conceituações de virtudes religiosas, perdão, altruísmo, esperança, prece e voluntarismo, apesar de soar operacional ou métrico, pode definir a nova direção para conduzir estudos de avaliação de espiritualidade e religiosidade.

Há tendência à correlação entre a religiosidade/espiritualidade e a saúde física, mas por ainda não ser adequadamente robusto em suas provas e correlações, este constitui, sem dúvida, em amplo e promissor campo de investigação. Nesse cenário, a necessidade de maior investigação da relação entre saúde física e espiritualidade, baseada principalmente no impacto de intervenções de base religiosa sobre a saúde, faz-se ainda relevante para a comprovação desse paradigma. A comprovação definitiva de efeitos dessas intervenções poderá, em futuro próximo, permitir sua transposição à prática clínica.

A utilização de adequado método científico e emprego dos princípios da medicina baseada em evidências, para avaliação crítica da literatura e a condução de estudos, pode certamente prover o caminho que moverá as hipóteses do promissor ao comprovado e certamente apenas essas confirmações poderão consolidar o paradigma suficiente para a modificação da percepção e conduta da sociedade atual ante a correlação entre espiritualidade e saúde.

\section{REFERÊNCIAS BIBLIOGRÁFICAS:}

Harris, T.B; Ferrucci, L.; Tracy R.P.; Corti, M.C.; Wacholder, S.; Ettinger, W.H. - Associations of elevated interleukin-6 and C-reactive protein levels with mortality in the elderly. American Journal of Medicine 106: 506512, 1999a. [ Links ]

Hummer, R.A.; Rodgers, R.G.; Nam, C.B.; Ellison, C.G. - Religious involvement and U.S. adult mortality. Demography 36(2): 273-285, $1999 . \quad$ [ Links ]

Ironson, G.; Stuetzie, R.; Flectcher, M.A. - An increase in religiousness/spirituality occurs after HIV diagnosis and predicts slower disease progression over 4 years in people with HIV. Journal of General Internal Medicine 21:S62-68, 2006.

Moreira-Almeida, A.L.; Lotufo Neto, F.; Koenig, H.G. - Religiousness and mental health: a review. Rev Bras Psiquiatr 28(3):242-50, 2006. [ Links ]

NHIS. Disponível em: www.pubmed.com. Acessado em 26 de março de 2007. [ Links ]

Oman, D.; Kurata, J. H., Strawbridge, W.J.; Cohen, R.D. - Religious attendance and cause of death over 31 years. International Journal of Psychiatry in Medicine 32:69-89, $2002 . \quad$ [ Links ]

Powell, L.H.; Shahabi, L.; Thoresen, C.E. - Religion and spirituality. Linkages to Physical Health. American

Psychologist 58(1):36-52, 2003. [ Links ]

Saad, M.; Masiero, D.; Battistella, L. - Espiritualidade baseada em evidências. Acta Fisiátrica 8(3):107-112, 2001. [ Links ]

Strawbridge, W.J.; Cohen, R.D.; Shema, S.J.; Kaplan G.A.K. - Frequent attendance at religious services and mortality over 28 years. Am J Public Health 87:957-961,1997. [ [ Links ]

Volcan, S.M.A. - Relationship between spiritual well-being and minor psychiatric disorders: a cross-sectional study. Rev. Saúde Pública 37(4):440-445, 2003. [ [ Links ] 


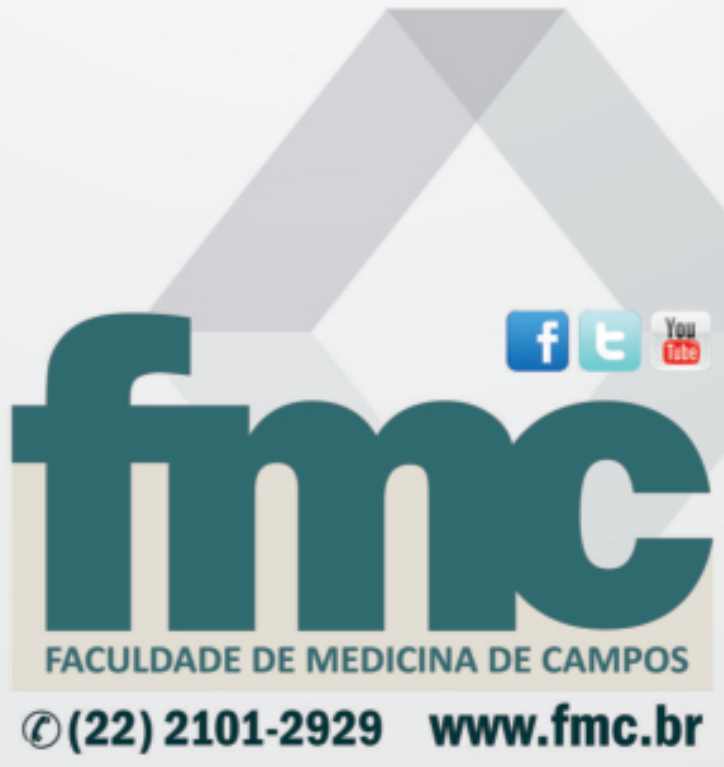

